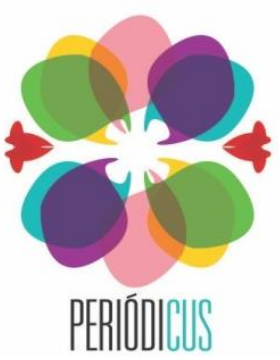

ISSN: 2358-0844

п. II, v. 2 mai. -out. 2019 р. $195-216$.

\title{
Transfobia:
}

\section{contextos de negatividade, violência e resistência}

\author{
João Paulo Zerbinati ${ }^{1}$ \\ Maria Alves de Toledo Bruns ${ }^{2}$
}

RESUMO: A transfobia é um fenômeno comum na vivência da população trans com consequências negativas para o seu bem-estar físico e psicológico, incluindo risco de homicídio e suicídio: uma questão de saúde pública, mental e de direitos humanos. A partir dessa agressiva realidade, esta revisão integrativa proporcionou uma síntese da literatura científica acerca dos fenômenos transfóbicos, seus contextos de violência e resistência à quebra da norma de sexo e gênero. Um total de 55 artigos atenderam os critérios de relevância do tema e foram incluídos nesta revisão. Os artigos foram analisados qualitativamente a partir de categorias de análise emergidas de suas contribuições, a saber: (1) "Transfobia e educação"; (2) "Transfobia e saúde"; (3) "Transfobia, raça e religião"; (4) "Transfobia e família; (5) "Transfobia adolescente"; (6) "Transfobia, psicologia e psicanálise"; (7) "Transfobia generalizada e transnegatividade internalizada". A violência transfóbica é o resultado das excludentes normas de gênero existentes e dos estereótipos mantidos pelos próprios mecanismos de controle sexual que os produziram. A transfobia está presente em grande parte das matrizes do viver trans, seja familiar, social, educacional, clínico, religioso, entre outras. Nesse sentido, ou o trans se enquadra aos modelos sexuais hegemônicos ou é jogado à margem da sociedade e mesmo se enquadrando ainda assim é excluído, pois inevitavelmente está fora dele. Diferentemente da agressão à pluralidade sexual e de gênero, que o impulso agressivo seja utilizado para transgredir e romper com a norma sexual binária, cisnormativa, naturalizante e excludente, frutificando posturas acolhedoras, mantenedoras e emancipatórias da vida, livres de estigma e transfobia.

PALAVRAS-CHAVE: transfobia; transnegatividade; intolerância; violência; revisão integrativa.

\begin{abstract}
Transphobia is a common phenomenon on the trans people life with negative consequences for their physical and psychological well-being, including the risk of homicide and suicide: a matter of public and mental health as well as human rights. Based on this aggressive reality, this integrative review provided a synthesis of the scientific literature on transphobic phenomena, their contexts of violence and resistance to the gender and gender norm disagreement. A total of 55 articles that met the relevance criteria of the theme were included. The articles were analyzed qualitatively from the analysis categories emanating from their contributions, being: (1) "Transphobia and education"; (2) "Transphobia and health"; (3) "Transphobia, race and religion"; (4) "Transphobia and family; (5) "Teen transphobia"; (6) "Transphobia, psychology and psychoanalysis"; (7) "Generalized transphobia and internalized transnegativity". Transphobic violence is the result of existing gender norms and sexual stereotypes maintained by mechanisms of sexual control that produced them. Transphobia is present in most part of the trans life context, being it family, social, educational, clinical, religious, among others. Being that way, the trans have to fit the hegemonics sexuals models or is put aside to the margins of the society. And even if they
\end{abstract}

${ }^{1}$ Mestre em Educação Sexual pela Faculdade de Ciências e Letras, UNESP/Araraquara. Membro do grupo de pesquisa SexualidadeVida-USP/CNPq. E-mail: joaopaulozerbinati@ hotmail.com

${ }^{2}$ Docente e Pesquisadora do Programa de Pós-graduação em Psicologia da Faculdade de Filosofia, Ciências e Letras da Universidade de São Paulo (USP-Ribeirão Preto) e do Programa de Pós-graduação em Educação Sexual da Faculdade de Ciências e Letras da Universidade Estadual Paulista (UNESP-Araraquara). Líder do Grupo de Pesquisa SexualidadeVida- USP/CNPq. E-mail: toledobruns@uol.com 
fits in, they ends up being excluded, because inevitably they are outside of models. Unlike the aggression to sexual and gender plurality, the aggressive impulse has to be used to transgress and break from the binary sexual norm, cisnormative, enabling welcoming and emancipatory postures of life, free from stigma and transphobia.

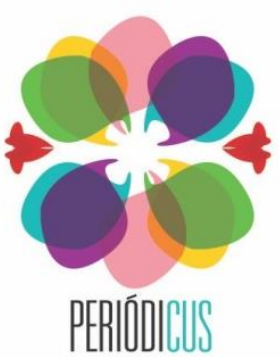

ISSN: 2358-7844 ก. II, v. 2 mai. -out. 2019 р. $195-216$.

Keywords: transphobia; transnegativity; intolerance; violence; integrative review.

Resumen: La transfobia es un fenómeno común en la vivencia de la población trans con consecuencias negativas para su bienestar físico y psicológico, incluyendo riesgo de homicidio y suicidio: una cuestión de salud pública, mental y de derechos humanos. A partir de esa agresiva realidad, esta revisión integrativa proporcionó una síntesis de la literatura científica acerca de los fenómenos transfóbicos, sus contextos de violencia y resistencia a la quiebra de la norma de sexo y género. Un total de 55 artículos atendieron los criterios de relevancia del tema y se incluyeron en esta revisión. Los productos se analizaron cualitativamente surgieron de categorias de análisis de sus contribuciones, a saber, (1) "Transphobia y educación"; (2) "Transfobia y salud"; (3) "Transfobia, raza y religión"; (4) "Transfobia y familia; (5) "Transfobia adolescente"; (6) "Transfobia, psicología y psicoanálisis"; (7) " Transfobia generalizada y transnegatividad internalizada". La violencia transfóbica es el resultado de las excluyentes normas de género existentes y de los estereotipos sexuales mantenidos por los propios mecanismos de control sexual que los produjeron. La transfobia está presente en gran parte de las matrices del vivir trans, sea familiar, social, educativo, clínico, religioso, entre otras. En ese sentido, o el trans se enmarca a los modelos sexuales hegemónicos o se juega al margen de la sociedad e incluso si encuadrando aún así es excluido, pues inevitablemente está fuera de él. De modo contrario a la agresión a la pluralidad sexual y de género, que el impulso agresivo sea utilizado para transgredir y romper con la norma sexual binaria, cisnormativas, naturalizante y excluyente, posibilitando posturas acogedoras, mantenedoras y emancipatorias de la vida, libres de estigma y transfobia.

Palabras clave: transfobia; transnegatividad; intolerância; violência; revisión integrativa.

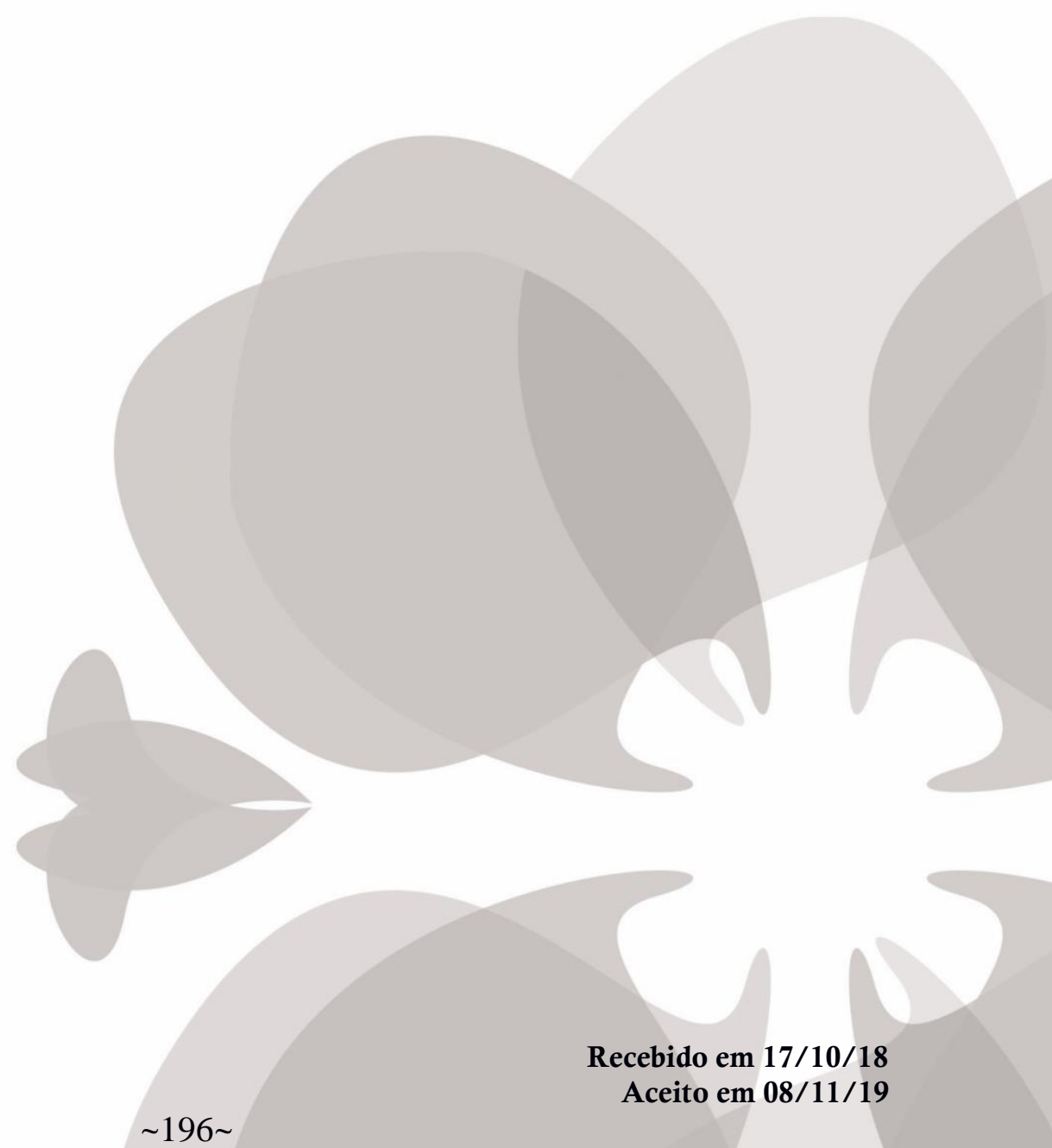




\section{Introdução}

A transexualidade, travestilidade, transgeneridade ou qualquer outra possibilidade de identidade de gênero que transite por entre as normas sexuais rígidas serão referidas neste artigo como identidades trans. Assim como considera Benedetti (2005), o termo trans possibilita ampliar as definições entorno do gênero, abrangendo as construções e desconstruções de seus próprios limites. O conceito trans vem ao encontro da proposta e esforço contemporâneo de não delimitar novas fronteiras entre as identidades de gênero (FRANCO; CICILLINI, 2015), buscando um termo o mais amplo e inclusivo possível (AMERICAN PSYCHOLOGICAL ASSOCIATION, 2015). Tais propostas terminológicas são importantes, pois promovem a inclusão e o acolhimento de todo o processo subjetivo de identidade sexual e de gênero, se distanciando de normatizações sexuais (ROCON; RODRIGUES; ZAMBONI; PEDRINI, 2016).

A sociedade, em âmbito global, está sendo intimada a discutir acerca das normas do gênero e sexo, muitas vezes repressoras, excludentes e violentas. No horizonte das diversidades sexuais e de gênero, as identidades trans se destacam devido à urgente demanda em ordem de saúde pública, educação, cultura e lazer, enfrentando, muitas vezes, posturas agressivas e violentas. Segundo o relatório de 2016 publicado pela ONG Transgender Eurupe (TGEu) ${ }^{3}$, entre 2008 e junho de 2016, 2.190 pessoas trans foram assassinadas em 66 países em todo o mundo. 78.1\% das mortes aconteceram na região da América Central e do Sul (incluindo o Caribe), 9\% no continente Asiático, 6.9\% na região da América do Norte, $5.2 \%$ no continente Europeu, $0.5 \%$ na África e $0.2 \%$ na Oceania.

Ainda segundo o relatório, os maiores índices absolutos foram encontrados em países com forte visibilidade e organização de comunidades LGBT+ (Lésbicas, Gays, Bissexuais, Travestis e Transexuais, entre outras diversidades afetivo-sexuais e de gênero). O Brasil é o país que mais mata pessoas trans no mundo, ao menos já foram 868 pessoas trans assassinadas entre os anos de 2008 e 2016. O Brasil é seguido pelo México (259), Colômbia (109), Venezuela (109) e Honduras (86), na América Central e do Sul; os Estados Unidos (146), na América do Norte, Turquia (43) e Itália (30), na Europa, assim como na Índia (58) e nas Filipinas (41), na Ásia.

3 “TMM annual report 2016”, disponível em <http://transrespect.org/wp-content/uploads/2016/11/TvT-PS-Vol142016.pdf>. Acesso em 11 out 2019.

Periódicus, Salvador, n.11, v. 2, mai-out.2019 - Revista de estudos indisciplinares em gêneros e sexualidades Publicação periódica vinculada ao Núcleo de Pesquisa NuCuS, da Universidade Federal da Bahia - UFBA ISSN: 2358-0844 - Endereço: http://www.portalseer.ufba.br/index.php/revistaperiodicus 
A violência letal contra minorias sexuais e de gênero não se trata de crimes comuns, decorridos de assalto, bala perdida ou crimes passionais, mas são "crimes de ódio". A condição sexual e de gênero da vítima foi "determinante no 'modus operandi' do agressor" (MOTT, 2006, p. 514). A agressividade dirigida às identidades trans pode ser definida como transfobia, um processo de recusa histórica, social e cultural do modo como a pessoa trans constrói sua identidade de gênero e sua sexualidade (FRANCO; CICILLINI, 2015, p.330). A transfobia se baseia em medo, desconforto, intolerância, rejeição, aversão, ódio, discriminação ou estigmatização à pessoa trans devido sua identidade de gênero (ORGANIZAÇÃO DAS NAÇÕES UNIDAS PARA A EDUCAÇÃO, A CIÊNCIA E A CULTURA, UNESCO, 2017).

O comportamento transfóbico acontece sob a forma de opiniões negativas, exclusão social/institucional, agressões físicas, verbais ou psicológicas manifestadas mesmo que indiretamente com a pretensão de reforçar e garantir o discurso binário e naturalizante ao gênero a partir do sexo biológico (ONU, 2014). Há ainda, na literatura científica, a presença do termo transnegatividade. Essa nomenclatura é utilizada, às vezes, como sinônimo de transfobia (CARROLL; MIZOCK, 2017) ou para destacar a internalização do preconceito por parte do próprio sujeito trans, levando-o à compreensão negativa de certos aspectos de sua condição de gênero (STAPLES, et al., 2017; JACKMAN; DOLEZAL; BOCKTING, 2017).

Vergueiro (2015, p.70) permite problematizarmos o conceito de transfobia. A autora propõe o conceito de cisheteronormatividade em uma perspectiva queer e decolonial e compreende a cisnormatividade e cissexismo enquanto "estruturantes de violência institucional, sociocultural, e existencialmente impregnadas por culturas e sociedades". Essas normas colocam as pessoas trans no patamar da não inteligibilidade, do estranhamento, impede-as de, assim como destacado por Zerbinati e Bruns (2018, p.80), "ser alguém em algum lugar", o que provoca sofrimento e o sentimento de não pertencimento.

A partir dessa agressiva realidade, que nos coloca questões de saúde pública, mental e de direitos humanos, é pertinente e necessário investigar as considerações científicas acerca da temática transfobia, quais as suas particularidades e delimitações em nível internacional. Esta revisão integrativa objetivou fornecer uma síntese da literatura acerca dos fenômenos transfóbicos, de cissexismo institucional, no intuito de elucidar e compreender seus contextos de violência e resistência à quebra da norma de sexo e gênero. 


\section{Método}

A revisão integrativa é um método de revisão que possibilita uma compreensão abrangente de um fenômeno particular (WHITTEMORE; KNAFL, 2005). Optamos pela realização da uma análise integrativa por ser uma estratégia de pesquisa detalhada, que proporciona uma síntese de conhecimento científico, incluindo estudos quantitativos e qualitativos (HOPIA; LATVALA; LIIMATAINEN, 2016; WHITTEMORE; KNAFL, 2005; SOUZA; SILVA; CARVALHO, 2010).

Esta revisão integrativa foi realizada de acordo com o método proposto por Souza; Silva e Carvalho (2010). Como estratégia metodológica, as autoras sugerem seis etapas para a elaboração de uma revisão integrativa: (1) elaboração da pergunta norteadora; (2) estratégia de busca, localização, definição de critérios de inclusão e exclusão; (3) coleta de dados e análise da qualidade dos estudos; (4) análise crítica dos estudos incluídos; (5) discussão dos resultados e (6) apresentação da revisão integrativa.

A busca na literatura para esta revisão foi realizada no período de janeiro de 2018, abrangendo três grandes bancos de dados eletrônicos, amplamente utilizados pelas ciências da saúde e humanas: SciELO (Scientific Electronic Library Online), Bireme (Biblioteca Regional de Medicina) e PubMed (National Library of Medicine). Para busca dos artigos foram utilizados os descritores "Transphobia" e "Transnegativity".

Todos os artigos completos disponíveis foram incluídos. Foram excluídos os artigos duplicados ou fora da área de interesse. Os artigos selecionados foram lidos na íntegra pelos autores de forma independente para confirmação quanto à pertinência para este estudo. Seus dados foram extraídos e inseridos em planilhas do Excel. A qualidade dos artigos foi determinada pelo seu rigor metodológico. A relevância do artigo foi determinada pelo foco em discutir aspectos transfóbicos ou transnegativos.

A análise consistiu na redução, exposição e comparação dos artigos (SOUZA; SILVA; CARVALHO, 2010). A fim de facilitar a comparação dos estudos, interpretações, sínteses e a discussão dos resultados, os artigos foram organizados em categorias emergidas a partir de seus principais resultados, podendo um artigo estar em mais de uma categoria ao mesmo tempo.

Periódicus, Salvador, n.11, v. 2, mai-out.2019 - Revista de estudos indisciplinares em gêneros e sexualidades Publicação periódica vinculada ao Núcleo de Pesquisa NuCuS, da Universidade Federal da Bahia - UFBA ISSN: 2358-0844 - Endereço: http://www.portalseer.ufba.br/index.php/revistaperiodicus 


\section{Resultados e discussão}

Foram identificados 161 artigos, obtidos a partir do PubMed $(n=77)$, Bireme $(n=73)$ e Scielo $(n=11)$. Com a retirada dos artigos duplicados e fora da área de interesse, relevância e qualidade, foram excluídos 106 artigos. O principal motivo para a exclusão dos artigos, após análise de texto completo pelos autores, foi a averiguação de que não investigavam diretamente nenhum aspecto transfóbico. Não houveram artigos incluídos na síntese qualitativa ou quantitativa, obtendo ao final uma amostragem de 55 artigos (Fig. 1).

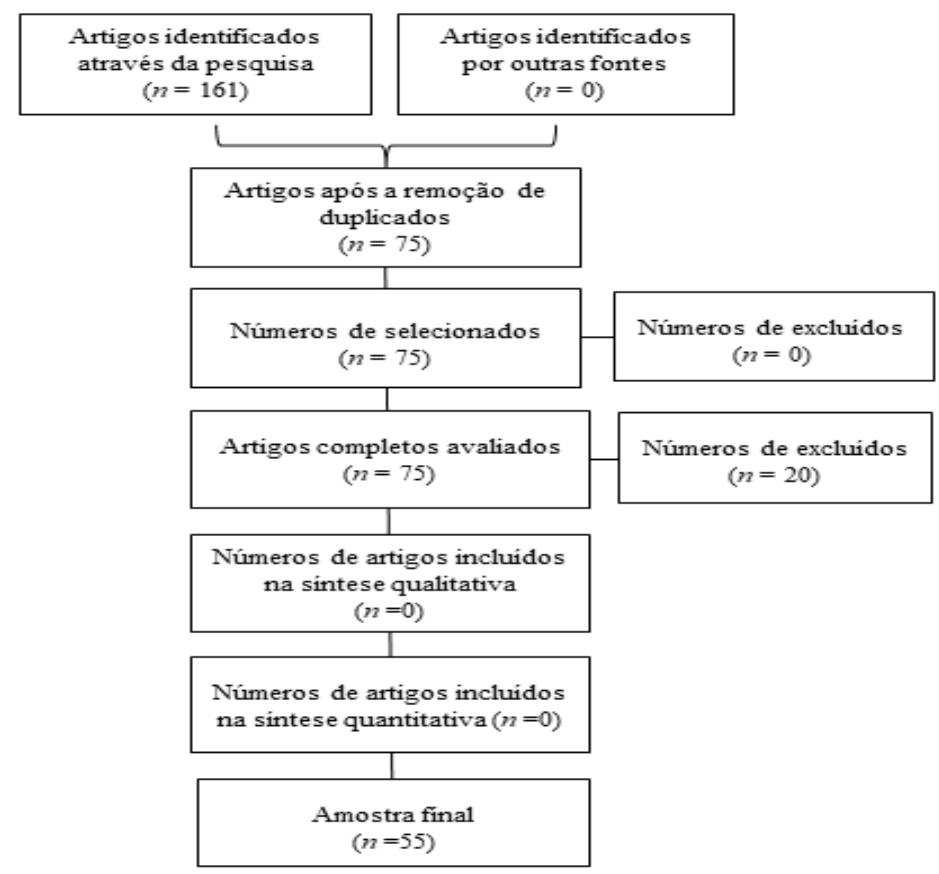

Fig. 1: Diagrama de fluxo de estudo (modelo prisma).

A Tab. 1 apresenta algumas das principais características dos artigos analisados, sendo possível averiguar que todos os artigos foram publicados entre 2002 e 2017. A maioria foi publicada em 2017 $(n=17)$ e $60 \%$ da amostra foi publicada nos últimos três anos $(n=33)$, o que demonstra a relevância e a contemporaneidade desse tema. A maioria dos artigos obtinha filiação a instituições dos Estados Unidos $(n=28)$. No entanto, Canadá $(n=10)$, Brasil $(n=3)$, Espanha $(n=3)$, Reino Unido $(n=3)$, África do Sul (2), Itália $(n=2)$, Austrália $(n=1)$, Bélgica $(n=1)$, Croácia $(n=1)$ e Portugal $(n=1)$ também foram representados. Desses, sete artigos teóricos e 48 empíricos. A maioria dos estudos empregaram métodos qualitativos $(n=30)$, embora estudos quantitativos $(n=20)$ e quanti/quali $(n=$ 5) também foram identificados. A maioria dos artigos obtinha como sujeito de estudo "pessoas trans

Periódicus, Salvador, n.11, v. 2, mai-out.2019 - Revista de estudos indisciplinares em gêneros e sexualidades Publicação periódica vinculada ao Núcleo de Pesquisa NuCuS, da Universidade Federal da Bahia - UFBA ISSN: 2358-0844 - Endereço: http://www.portalseer.ufba.br/index.php/revistaperiodicus 
indiscriminadas" $(n=21)$, seguido por artigos acerca de "mulheres trans" $(n=15)$, "jovens trans" $(n=$ 4), "homens trans" $(n=1)$, "professoras trans" $(n=1)$, "pais trans" $(n=1)$, "parceiros de trans" $(n=2)$, "estudantes universitários cis" $(n=3)$, "adolescentes cis" $(n=3)$, "população geral cis" $(n=1)$ e "médicos cis" $(n=3)$. Aproximadamente $78 \%$ das investigações são acerca de sujeitos trans e 22\% referentes aos sujeitos cisgêneros ${ }^{4}$.

Os artigos selecionados foram organizados em sete categorias de análises (Tab. 2), possibilitando a análise qualitativa no intuito de resumir as descobertas e fornecer recomendações para o trabalho em amplo campo de possibilidades clínicas, educacionais e institucionais, levando em consideração a compreensão dos aspectos transfóbicos.

\footnotetext{
${ }^{4}$ Cisgênero ou "cis" é um termo utilizado para se referir às pessoas que estão em total sintonia com a identidade de
} gênero lhes atribuída no nascimento, a partir do sexo biológico.

Periódicus, Salvador, n.11, v. 2, mai-out.2019 - Revista de estudos indisciplinares em gêneros e sexualidades Publicação periódica vinculada ao Núcleo de Pesquisa NuCuS, da Universidade Federal da Bahia - UFBA ISSN: 2358-0844 - Endereço: http://www.portalseer.ufba.br/index.php/revistaperiodicus 
Tab. 1: Características dos estudos analisados.

\begin{tabular}{|c|c|c|c|c|c|c|c|c|c|}
\hline \multicolumn{2}{|c|}{ Ano de publicação } & \multicolumn{2}{|c|}{ País de origem } & \multicolumn{2}{|c|}{ Tipo de estudo } & \multicolumn{2}{|c|}{ Tipo de análise } & \multicolumn{2}{|l|}{ Público alvo } \\
\hline Categorias & $n(\%)$ & Categorias & $n(\%)$ & Categorias & $n(\%)$ & Categorias & $n(\%)$ & Categorias & $n(\%)$ \\
\hline 2002 & $1(1.8)$ & EUA & $28(51.0)$ & Empírico & $48(87.3)$ & Qualitativa & $30(54,5)$ & Pessoas trans & $21(38.2)$ \\
\hline 2004 & $1(1.8)$ & Canadá & $10(18.2)$ & Teórico & $7(12.7)$ & Quantitativa & $20(36.4)$ & Mulheres trans & $15(27.3)$ \\
\hline 2006 & $2(3.6)$ & Brasil & $3(5.4)$ & & & Quanti/Quali & $5(9.1)$ & Jovens trans & $4(7.3)$ \\
\hline 2007 & $1(1.8)$ & Espanha & $3(5.4)$ & & & & & Homens trans & $1(1.8)$ \\
\hline 2009 & $1(1.8)$ & Reino Unido & $3(5.4)$ & & & & & Professoras trans & $1(1.8)$ \\
\hline 2011 & $5(9.1)$ & África do Sul & $2(3.6)$ & & & & & Pais trans & $1(1.8)$ \\
\hline 2012 & $6(11.0)$ & Itália & $2(3.6)$ & & & & & Parceiros de trans & $2(3.7)$ \\
\hline 2013 & $2(3.6)$ & Austrália & $1(1.8)$ & & & & & Universitários cis & $3(5.4)$ \\
\hline 2014 & $3(5.4)$ & Bélgica & $1(1.8)$ & & & & & Adolescentes cis & $3(5.4)$ \\
\hline 2015 & $11(20.0)$ & Croácia & $1(1.8)$ & & & & & População cis & $1(1.8)$ \\
\hline 2016 & $5(9.1)$ & Portugal & $1(1.8)$ & & & & & Médicos cis & $3(5.4)$ \\
\hline 2017 & $17(31.0)$ & & & & & & & & \\
\hline
\end{tabular}

Periódicus, Salvador, n.11, v. 2, mai-out.2019 - Revista de estudos indisciplinares em gêneros e sexualidades 
Tab. 2: Categorização dos artigos selecionados para análise $(n=55)$.

\begin{tabular}{|c|c|c|}
\hline Categorias de análise & Descrição & Referências \\
\hline $\begin{array}{l}\text { Transfobia e Educação } \\
(n=9)\end{array}$ & $\begin{array}{l}\text { Artigos que discutem a transfobia no âmbito } \\
\text { educacional. }\end{array}$ & $\begin{array}{l}\text { LOVAAS; BAROUDI; COLLINS (2002); SAUSA; KEATLEY; OPERARIO (2007); TAYLOR; PETER (2011); } \\
\text { CALDAS, et al. (2012); FRANCO; CICILLINI (2015); WEINER; ZINNER (2015); ALI; FLEISHER; ERICKSON } \\
\text { (2016); ACKER (2017); DEVÍS-DEVÍS et al. (2017) }\end{array}$ \\
\hline $\begin{array}{l}\text { Transfobia e Saúde }(n= \\
\text { 20) }\end{array}$ & $\begin{array}{l}\text { Investigações acerca da transfobia na saúde } \\
\text { pública, nas instituições de saúde e dos } \\
\text { profissionais de saúde. }\end{array}$ & $\begin{array}{l}\text { NEMOTO, et al. (2004); SUGANO; NEMOTO; OPERARIO (2006); DALEY; MACDONNELL (2011); LOGIE, } \\
\text { et al. (2011); CHAPMAN, et al. (2012); LOGIE, et al. (2012); NEMOTO et al. (2012); KOSENKO, et al. } \\
\text { (2013); NEWMAN-VALENTINE; DUMA (2014); ESCUDERO et al. (2015); LACOMBE-DUNCAN (2016); } \\
\text { LEVITT (2015); LYONS et al. (2015); MCPHAIL; ROUNTREE-JAMES; WHETTER (2016); SEVELIUS, et al. } \\
\text { (2016); ABRAMOVICH (2017); BRAUN et al. (2017); HOFFKLING; OBEDIN-MALIVER; SEVELIUS (2017); } \\
\text { MÜLLER (2017); O'NEILL; WAKEFIELD (2017). }\end{array}$ \\
\hline $\begin{array}{l}\text { Transfobia, psicologia e } \\
\text { psicanálise }(n=2)\end{array}$ & $\begin{array}{l}\text { Discute criticamente a transfobia na } \\
\text { psicologia e psicanálise. }\end{array}$ & AMODEO et al. (2015); BOLAND (2017). \\
\hline $\begin{array}{l}\text { Transfobia, raça e religião } \\
(n=3)\end{array}$ & $\begin{array}{l}\text { Artigos que relacionaram a transfobia à raça } \\
\text { e/ou religião. }\end{array}$ & DALEY; MACDONNELL (2011); FERNANDES (2013); FISHER et al (2017). \\
\hline Transfobia e Família $(n=4)$ & $\begin{array}{l}\text { Pesquisas sobre a transfobia familiar, seus } \\
\text { fatores de risco e proteção. }\end{array}$ & $\begin{array}{l}\text { BORNSTEIN ET AL (2006); JOKIĆ-BEGIĆ; KORAJLIJA; JURIN (2014); KAPLAN et al. (2015); MUÑOZ- } \\
\text { LABOY, et al. (2017). }\end{array}$ \\
\hline $\begin{array}{l}\text { Transfobia adolescente } \\
(n=7) \text {. }\end{array}$ & $\begin{array}{l}\text { Investiga a transfobia oriunda de adolescentes } \\
\text { cis }\end{array}$ & $\begin{array}{l}\text { IANTAFFI; BOCKTING (2011); CALDAS, et al. (2012); COSTA; DAVIES (2012); TEBBE; BONNIE (2012); } \\
\text { CARRERA-FERNÁNDEZ et al. (2014); OTERO et al. (2015); MUÑOZ-LABOY, et al. (2017). }\end{array}$ \\
\hline $\begin{array}{l}\text { Transfobia generalizada y } \\
\text { transnegatividad } \\
\text { internalizada }(n=13)\end{array}$ & $\begin{array}{l}\text { Artigos focalizados em compreender a } \\
\text { transfobia socialmente generalizada e sua } \\
\text { relação com a transnegatividade internalizada } \\
\text { por sujeitos trans. }\end{array}$ & $\begin{array}{l}\text { LOMBARDI (2009); NEMOTO; BÖDEKER; IWAMOTO (2011); BAUER ET AL. (2015); CLAES et al. (2015); } \\
\text { PEREZ-BRUMER, et al. (2015); ARCELUS et al. (2016); AUSTIN; GOODMAN (2017); CALLAHAN; } \\
\text { ZUKOWSKI (2017); JACKMAN; DOLEZAL; BOCKTING (2017); LYONS, et al. (2017); MCDERMOTT; } \\
\text { HUGHES; RAWLINGS (2017); STAPLES, et al. (2017); TESTA el al. (2017). }\end{array}$ \\
\hline
\end{tabular}




\subsection{Transfobia e educação}

Franco e Cicillini (2015) denunciam que um dos principais cenários de estigma, preconceito e exclusão às pessoas trans acontece nas escolas e universidades. Corroborando com esse dado, Devís-devís et al. (2017), em um estudo para identificar o padrão de assédio e perfil de risco em pessoas trans espanholas, mostraram que aproximadamente $46 \%$ dos assédios e ataques verbais aconteceram em contextos educacionais.

Os estudantes refletem o ambiente transfóbico/cissexista em que vivem e possuem um entendimento preconceituoso da identidade trans, comumente atribuindo significados pejorativos, de anormalidade, perversão e psicopatologia às pessoas que transgridem, em algum sentido, os modelos heteronormativos e cisheteronoemativos ${ }^{1}$ (CALDAS, et al., 2012; WEINER; ZINNER, 2015). Em outro estudo com universitários, Acker (2017) demonstra que quase metade dos alunos apresentaram níveis moderados ou altos de transfobia.

No contexto educacional, a violência, seja velada ou materializada em agressões físicas dirigidas às identidades trans são desencadeadas não apenas por alunos, mas também pelo corpo docente. Essa contínua exposição à violência, além de prejudicar o aluno fora dos padrões sexuais hegemônicos, fere seus direitos e contribui para sua evasão escolar. Em decorrência do ambiente hostil, poucos sujeitos trans conseguem concluir os estudos básicos. Por esse motivo, acabam se aproximando de contextos de vulnerabilidade social e comportamentos de risco, como uso abusivo de drogas e prostituição desprovida de práticas de cuidado com a saúde (SAUSA; KEATLEY; OPERARIO, 2007; TAYLOR; PETER, 2011; FRANCO; CICILIINI, 2015).

No intuito de amenizar os processos de exclusão e violência, algumas pessoas trans acabam desenvolvendo rendimentos escolares acima da média. A partir desse caminho, alguns/mas trans

\footnotetext{
${ }^{1}$ Heteronormatividade pode ser descrita como a crença de que a heterossexualidade é o modelo ideal e que tod as as outras orientações afetivo-sexuais são inferiores. A partir dessa lógica, as estruturas sociais são concebidas e reforçadas de modo a acomodar a heterossexualidade, negando direitos e status de normalidade às orientações afetivo-sexuais não heterossexuais (PENNINGTON; KNIGHT, 2011). Essa crença é fruto do que Butler (1990/2003) chamou de heteronormatividade compulsória, que aprisiona e exclui o sexualmente diverso, uma lógica dominante da heterossexualidade que se institui e se reitera a partir da ordem compulsória do sexo/gênero/d esejo, estabelecendo um padrão de "normalidade", que vai produzir efeitos sociais de hierarquia, classificação, dominação e exclusão. Nesse sentido, vemos como adequado ampliar o termo heteronormativo de modo a englobar também a cisnormatividade, tal como proposto por Vergueiro (2015) e Jardim (2016), destacando à determinação e naturalização da existência de uma coerência entre o sexo de nascimento e a identidade de gênero, considerando a constituição de gênero cisgênero como 'padrão natural'.
}

Periódicus, Salvador, n.11, v. 2, mai-out.2019 - Revista de estudos indisciplinares em gêneros e sexualidades Publicação periódica vinculada ao Núcleo de Pesquisa NuCuS, da Universidade Federal da Bahia - UFBA ISSN: 2358-0844 - Endereço: http://www.portalseer.ufba.br/index.php/revistaperiodicus 
motivam-se à carreira docente como uma maneira de se proteger do "universo masculino" comumente agressivo e violento aos que fora da norma cisheterossexual se posicionam e por perceberem a educação como um importante instrumento para mudanças (FRANCO; CICILLINI, 2015, p.340).

A transfobia na educação demonstra a carência de conteúdos acadêmicos e educacionais direcionados à sexualidade. A literatura científica destaca a necessidade de disciplinas, materiais didáticos e palestras com o objetivo de oferecer aos alunos o conhecimento adequado e especializado sobre a sexualidade. A reflexão acerca da diversidade sexual e de gênero deve ser incorporada transversalmente ao longo dos diferentes ciclos educacionais, da escola à universidade e educação continuada, para uma educação igualitária a todos, independente do gênero, sexo ou orientação afetivosexual (CALDAS, et al., 2012; FRANCO; CICILLINI, 2015; ACKER, 2017).

Todas essas vivências e experimentações descritas nas narrativas dos sujeitos da pesquisa destacaram uma necessidade de reestruturação dos princípios norteadores da educação. Isto é, como formas dissidentes de construção do gênero e vivência das sexualidades são compreendidas nesse espaço que se reafirma constantemente como o local do aprendizado e vivência da normatização, muitas vezes impostas por distintas variações de violência (FRANCO; CICILLINI, 2015, p.336).

Lovaas; Baroudi e Collins (2002) propõem a utilização da teoria queer como uma estratégia pedagógica crítica e propícia ao diálogo e desconstrução de posturas LGBTfóbicas/cissexistas. Projetos educacionais amparados em uma abordagem inclusiva das diversidades afetivo-sexuais, potentes à promoção de mudanças discursivas acerca da sexualidade, tal como a teoria queer, tendem a resultar no aumento da compreensão e inclusão à população trans, uma vez que o discurso LGBTfóbico/cissexista parece estar relacionado a equivocados entendimentos cientificistas, religiosos e populares acerca do sexo e do gênero, assim como ao pouco contato interpessoal com pessoas trans (ALI; FLEISHER; ERICKSON, 2016).

\subsection{Transfobia e saúde}

A exposição a um ambiente cissexista na infância e adolescência está relacionada a impactos negativos na saúde dos jovens trans, pois leva a comportamentos de risco, como uso abusivo de substâncias psicoativas ilícitas e comportamento sexual não seguro (NEMOTO et al., 2012; NEMOTO, et al., 2004; SUGANO, NEMOTO; OPERARIO, 2006). Além disso, a transfobia presente nas instituições de saúde interfere direta e negativamente no cuidado com a saúde das pessoas trans (DALEY; MACDONNELL, 2011; KOSENKO, et al., 2013; NEWMAN-VALENTINE; DUMA, 2014;

Periódicus, Salvador, n.11, v. 2, mai-out.2019 - Revista de estudos indisciplinares em gêneros e sexualidades Publicação periódica vinculada ao Núcleo de Pesquisa NuCuS, da Universidade Federal da Bahia - UFBA ISSN: 2358-0844 - Endereço: http://www.portalseer.ufba.br/index.php/revistaperiodicus 
MCPHAIL; ROUNTREE-JAMES; WHETTER, 2016; SEVELIUS, et al., 2016).

Müller (2017) analisa experiências de pessoas trans e LGBTs em serviços de saúde pública na África do Sul e discute a prevalência absoluta de experiências discriminatórias pelos profissionais de saúde em relação à orientação afetivo-sexual e/ou identidade de gênero dos/as pacientes. Aos/às jovens LGBTs são criados obstáculos ao acesso de serviços de suporte à saúde. Alguns/mas jovens chegaram ao ponto de terem o direito ao serviço de saúde negado.

O que parece existir é algo como uma promulgação sistêmica de LGBTfobia (ABRAMOVICH, 2017). O preconceito e maus tratos nas instituições de saúde são barreiras que dificultam o cuidado à saúde de pessoas trans nos mais diferentes níveis de atenção à saúde, do tratamento por uso abusivo de substâncias (LYONS et al. 2015) ao tratamento de câncer (LEVITT, 2015) e tratamento do HIV (LACOMBE-DUNCAN, 2016; ESCUDERO et al, 2015; LOGIE, et a., (2012).

Desvelar posturas cissexistas na saúde é um importante passo no sentido de assinalar os efeitos deletérios que o estigma, preconceito e discriminação provocam à saúde trans e orientar práticas de tratamento, cuidado e suporte à saúde que possam acolher e incluir demandas específicas da população trans (LOGIE, et al., 2011). Por exemplo, Sevelius, et al. (2016) sublinham a importância de retirar as mulheres trans do grupo de HSH (homens que fazem sexo com homens) como estratégia para prevenção ao HIV. Enquadrar as mulheres trans no grupo de 'homens' é entendido como transfóbico e abusivo, pois vai contra sua identidade de gênero, sua história de vida. Esse ataque ao reconhecimento de seu gênero acaba afastando as mulheres trans de abordagens terapêuticas como a PrEP (Profilaxia pré-Exposição).

Adequações básicas nos setores de cuidado à saúde podem trazer resultados positivos à saúde das pessoas trans. Assim, é significativo atentar-se a alguns importantes aspectos como: utilizar de linguagem inclusiva para todos/as os/as pacientes; não fazer suposições acerca da identidade de gênero e orientação sexual; incluir demandas trans em cartilhas, cartazes e demais materiais educativos, seja destinado à população ou aos profissionais da saúde. O ambiente de saúde deve ser inclusivo aos/às pacientes trans e seus familiares no intuito de potencializar a escuta, o acolhimento, logo o próprio cuidado (O’NEILL; WAKEFIELD, 2017).

Outra importante questão a ser discutida pela saúde é o processo de planejamento reprodutivo (concepção, gravidez, parto e pós-parto) de homens trans, suas necessidades e dificuldades. A transfobia é atribuída como o maior desafio ao cuidado com a saúde de homens trans que engravidam. Alguns 
homens trans acabam por não realizar o adequado acompanhamento médico, outros se travestem de mulher na tentativa de minimizar o risco de vivências transfóbicas nesse período delicado (HOFFKLING, et al. 2017; SEVELIUS, et al., 2016).

No fenômeno de trânsito ao gênero binário, a gravidez de homens trans merece uma explanação maior, pois quebra com a máxima do discurso sexual e gênero pautado em uma lógica naturalizante. A lógica de uma coerência entre o sexo de nascimento, a identidade de gênero e a orientação afetivosexual, não legitima que identidades fora dessa coerência possam existir e ocupar posições até então fixadas como naturais a este ou aquele sexo/gênero binário (BUTLER, 1990/2003). Para se pensar no cuidado aos homens trans grávidos, assim como qualquer outra demanda trans, é preciso que ocorra a desconstrução dessas barreiras ideológica que demarcam papéis rígidos aos gêneros a partir do sexo de nascimento, como, por exemplo, a crença de que apenas mulheres cis engravidam por correlacionar vagina e útero ao gênero feminino.

Homens trans com vagina e útero também podem engravidar e estão engravidando! A questão da não-intelegibilidade é então tomada como a chave para compreensão da transfobia/cissexismo. Assim como compreende Butler (2014, p.253): "gênero é o mecanismo pelo qual as noções de masculino e feminino são produzidas e naturalizadas, mas gênero pode muito bem ser o aparato através do qual esses termos possam ser descontruídos e desnaturalizados". Para a real compreensão, cuidado e aceitação popular das identidades trans é necessário que os discursos que enrijecem e naturalizam a sexualidade sejam descontruídos inclusive dentro das instituições de saúde.

Muitos profissionais da saúde não estão habilitados para atender a demanda trans, assim como não possuem conhecimento necessário para o cuidado inclusivo. A falta de conhecimento e preparo leva à violência psicológica e mesmo física às pessoas trans por profissionais da saúde. Situações de maus tratos, sutis ou abertos, provocam distanciamento e desconfiança aos serviços de saúde por parte da população trans (CHAPMAN, et al., 2012; MÜLLER, 2017).

Braun, et al. (2017) avaliaram a oferta de cursos e disciplinas aos/às alunos/as das áreas da saúde a oportunidade para um melhor conhecimento quanto às especificidades na atenção à saúde das pessoas trans. Após o oferecimento de um curso eletivo para estudantes sobre a saúde trans, os escores imediatamente após o curso demonstraram um maior conhecimento e uma redução significativa de compreensões preconceituosas. Essa experiência levou os autores a concluírem que o oferecimento de conhecimento adequado e atualizado acerca da sexualidade e gênero pode trazer mudanças positivas, em um curto prazo, em relação ao aumento no domínio do conhecimento e redução de transfobia entre

Periódicus, Salvador, n.11, v. 2, mai-out.2019 - Revista de estudos indisciplinares em gêneros e sexualidades Publicação periódica vinculada ao Núcleo de Pesquisa NuCuS, da Universidade Federal da Bahia - UFBA ISSN: 2358-0844 - Endereço: http://www.portalseer.ufba.br/index.php/revistaperiodicus 
estudantes das áreas da saúde.

\subsection{Transfobia, psicologia e psicanálise}

A partir da importante função que o apoio emocional e de saúde mental pode desempenhar às pessoas trans, Boland (2017) discute as implicações do/a profissional da psicanálise para o acompanhamento psicológico de pacientes trans. Segundo a autora, para que o/a analista consiga desempenhar sua função, ele/a deve ter a capacidade de atentar-se aos desencadeadores contratransferenciais, perceber as implicações culturais e pessoais produzidas por uma cultura binária, para não cair em uma análise psicopatologizante, que venha invisibilizar as pessoas trans, deixando de compreender e reconhecer seu sofrimento e sua história.

Algumas vertentes psicanalíticas se arriscam na construção de espaços reflexivos, teórico e clínicos, não normativos sexualmente. Esse movimento importante pode criar novos espaços clínicos e institucionais de promoção de saúde mental e qualidade de vida, a partir da desconstrução interna do cissexismo e da cisheteronormatividade. Para isso, é preciso distanciar-se de um enquadre excludente e patologizante à diversidade afetivo-sexual e de gênero, infértil a qualquer possibilidade para um acompanhamento analítico ligado à promoção da saúde mental e desenvolvimento emocional (AMODEO et al. 2015).

\subsection{Transfobia, raça e religião}

Fernandes (2013) discorre acerca da relação entre a religião, raça, transfobia e homofobia de camadas pobres brasileiras. O autor correlaciona o fundamentalismo religioso aos atos de violência contra trans e gays negros/as ligados/as às religiões afro-brasileiras. Nesse mesmo sentido, Fisher et al. (2017) associa os preceitos e dogmas religiosos à homofobia e transfobia.

A relação direta entre a sexualidade e o pecado, feita por algumas religiões, são desencadeadoras de intolerância e agressividade à diversidade sexual e de gênero. O discurso religioso acaba por reforçar os estereótipos naturalizantes e binários do gênero e da sexualidade (DALEY; MACDONNELL, 2011).

\subsection{Transfobia e família}

A família é compreendida como um importante fator de proteção quando apresenta um discurso compreensivo e acolhedor da diversidade de gênero. $\mathrm{O}$ apoio familiar e social para a pessoa trans afeta 
de modo positivo sua autoestima, qualidade de vida e na saúde, incluindo saúde mental. O apoio familiar leva a uma melhor relação da pessoa trans com aspectos sociais, emocionais e financeiros (JOKIĆBEGIĆ; KORAJLIJA; JURIN, 2014; KAPLAN et al., 2015).

Muñoz-laboy, et al. (2017) demonstram que a transfobia também está presente nos relacionamentos afetivo-sexuais das pessoas trans. A violência doméstica afeta as pessoas trans de modo marcante, chegando a $80 \%$ dos casos. As mulheres trans são as mais afetadas por essa violência e o parceiro agressor normalmente é uma pessoa cisgênera.

Segundo Bornstein et al. (2006), sobreviventes de violência doméstica relataram dificuldade em identificar o comportamento de seus parceiros como abusivo. Há uma marca cultural em responsabilizar a vítima pelo abuso e/ou violência sofrida que acaba protegendo o agressor e dificultando o cuidado e atenção psicossocial à vítima. Os autores enfatizam a importância da educação sexual e informação nos serviços de atenção aos/às sobreviventes de violência doméstica, assim como educação sexual na comunidade para abordar e prevenir a violência doméstica.

\subsection{Transfobia adolescente}

Caldas, et al. (2012) discutem a influência que representações sexistas familiares têm nas posturas LGBTfóbicas dos/as filhos/as, levando a compreensões inadequadas e, às vezes, violentas. Uma sociedade transfóbica, sem oportunidade para se educar sexualmente, forma uma população transfóbica e machista: "a verdade é que os jovens ainda são educados de maneira sexista e homofóbica" (idem, p.153).

Costa e Davies (2012) revelaram que os adolescentes cis portugueses tendem a posicionamentos mais negativos que as adolescentes cis quanto à diversidade sexual. Em investigação parecida, Carrerafernández et al. (2014) avaliaram o posicionamento de adolescente cis espanhóis em relação às pessoas trans e também compreenderam que os meninos exibiram atitudes significativamente mais negativas às pessoas trans do que as meninas. Além disso, as mulheres trans são o principal alvo de opiniões transfóbicas emitidas por adolescentes cis (OTERO et al, 2015; MUÑOZ-LABOY, et al., 2017).

A relação da transfobia adolescente está diretamente associada ao ensino informal tradicional acerca dos papéis de gênero. A lógica binária e naturalizante do gênero cria o estigma à identidade de gênero trans. De modo contrário, crenças de gênero menos rígidas estão associadas a níveis mais baixos de transfobia e transfobia internalizada, que, por sua vez, estão associados a níveis mais 
elevados de autoestima e qualidade de vida na população trans (IANTAFFI; BOCKTING, 2011; TEBBE; BONNIE, 2012).

\subsection{Transfobia generalizada e transnegatividade internalizada}

A transfobia e cissexismo institucional se apresentam em diferentes níveis e contextos, são agressões que trazem sofrimento, problemas emocionais, de saúde, educacionais e sociais. É uma violência generalizada, estrutural e cultural que perpassa pela escola, família, instituições religiosas e chega a interferir na qualidade dos serviços prestados à população trans, seja de educação, saúde ou segurança pública (LYONS, et al., 2017).

A experiência transfóbica social e estrutural impacta profundamente a vivência das pessoas trans (LOMBARDI, 2009). A transfobia e a falta de apoio social foram correlacionadas ao suicídio, ideação suicida ou auto-mutilação não suicida, independente de doenças mentais como a depressão (NEMOTO; BÖDEKER; IWAMOTO, 2011; MCDERMOTT; HUGHES; RAWLINGS, 2017; STAPLES, et al., 2017).

Por detrás desse sintoma social está o silenciamento e a falta de educação sexual. Não falar sobre a transfobia/cissexismo ou, em última análise, sobre a própria sexualidade, dentro ou fora da norma sexual e de gênero binária, impede avanços compreensivos e dificulta o desenvolvimento emocional, profissional e a continuidade da vida de muitas pessoas trans (MCDERMOTT; HUGHES; RAWLINGS, 2017).

O risco elevado de auto-lesão não suicida e ideação suicida da população trans estão diretamente relacionados à transnegatividade internalizada (TESTA el al., 2017; ARCELUS et al., 2016; PEREZBRUMER, et al., 2015; BAUER et al., 2015; CLAES et al., 2015). De modo geral, as pesquisas sugerem que as atribuições negativas à identidade trans são internalizadas pelas pessoas trans e atuam como mediadores e moderadores desses fatores de risco (STAPLES et al., 2017), assim como impactam negativamente na auto-estima (AUSTIN; GOODMAN, 2017).

Ao comparar a transnegativiade internalizada entre jovens trans e um grupo trans de uma geração mais antiga nos Estados Unidos, Jackman; Dolezal e Bockting (2017) demonstraram que o grupo mais antigo obtinha níveis significativamente mais baixos de transnegatividade internalizada e sofrimento psicológico em comparação ao grupo mais jovem. Segundo o estudo, apesar de uma atual maior visibilidade das pessoas trans e da crescente aceitação da diversidade de gênero, as mulheres 
trans mais jovens relataram um maior sofrimento emocional que as mulheres trans mais velhas.

Jackman; Dolezal e Bockting (2017) consideram que esses achados estão, em parte, relacionados à transnegatividade internalizada, por conta da diferença da transfobia sofrida no passado em comparação à agressividade, por vezes desumana, da contemporaneidade. Além disso, os autores também sugerem que, com o passar dos anos, as trans mulheres acabam desenvolvendo uma maior resiliência à transfobia.

No tocante ao entendimento popular quanto à utilização de banheiros públicos por pessoas trans, Callahan e Zukowski (2017) estudaram as reações de pessoas cis quanto à utilização de banheiros públicos por pessoas trans. A opinião prevalente foi a de que os sujeitos trans devem usar os banheiros destinados ao seu gênero atribuído no nascimento. Entretanto reações negativas foram direcionadas aos/às trans independente do banheiro utilizado, seja o banheiro ao gênero que se identificam ou o banheiro correspondente ao gênero lhes atribuído no nascimento. A mensagem social incansavelmente enviada à população trans é a de que não há espaço para uma identidade de trânsito ao gênero e sexo.

Para que se possa combater a transfobia e a transfobia internalizada é preciso investir em estratégias que auxiliem a sociedade, assim como as próprias pessoas trans, na quebra dos inevitáveis preconceitos à identidade sexual e de gênero produzidos por uma sociedade machista e cissexista (PEREZ-BRUMER, et al., 2015; TESTA el al., 2017; ARCELUS et al., 2016).

\section{Considerações finais: horizontes de compreensão}

A partir dos dados aqui colhidos foi possível compreender que, seja no ambiente familiar, social, religioso, educacional ou de saúde, a lógica cisheteronormatividade representa um poder de controle sexual categórico, inflexível e violento. Nesse sentido, ou a pessoa trans se enquadra à norma sexual vigente ou é excluída e, mesmo se enquadrando, ainda assim é excluída, pois inevitavelmente está fora dela.

A identidade trans se constitui de um modo menos enrijecido, com potencial criativo e genuíno ao sexo e ao gênero. Uma sociedade cisheteronormativa e cissexista lida com a criação sexual e de gênero de modo agressivo por entender as pessoas trans como uma ameaça quando, na verdade, elas poderiam ser entendidas como defensoras de uma 'carta de alforria' às normas sexuais e de gênero para todos e todas. 
A violência transfóbica/cissexista é o resultado das normas sexuais e de gênero existentes e de seus estereótipos, reforçados pelos próprios poderes institucionais e cientificistas que os produziram. Nesse sentido, a educação sexual e de gênero pode ser um importante instrumento para promover discursos compreensivos e acolhedores da diversidade sexual e de gênero, atuando por meio da produção e popularização do conhecimento científico humano, intervindo diretamente em equivocados e preconceituosos modelos discursivos.

A educação sexual e de gênero é uma afetuosa e potente estratégia contra a transfobia e cissexismo. Diferentemente da agressão ao viver trans, que o impulso agressivo possa ocupar espaços para destruição da norma ao sexo e ao gênero, excludente, assassina e violenta. Que o conhecimento possa promover posturas humanas, éticas, acolhedoras, mantenedoras e emancipatórias da vida.

Esta revisão integrativa teve o objetivo de investigar e organizar as produções científicas acerca da transfobia e/ou transnegatividade de modo a oferecer uma importante contribuição para a compreensão da transfobia. É necessário e urgente que mais pesquisas com esse mesmo foco sejam produzidas, principalmente nos países com maior histórico de transfobia. Mais que isso, que esses estudos cheguem às camadas populares para sérias discussões nas escolas, nas universidades, nas famílias, nas igrejas, nas instituições de promoção à saúde, assim como quaisquer outros contextos de vivência humana, frutificando em compreensões inclusivas à diversidade sexual e de gênero e interventivas às posturas transfóbicas/cissexistas, assim como quaisquer desencadeadores violentos ao viver.

\section{Referências}

ABRAMOVICH, Alex. Understanding how policy and culture create oppressive conditions for LGBTQ2S youth in the shelter system. Journal of Homosexuality, v. 64, n. 11, p.1484-1501, 2017.

ACKER, Gila M. Transphobia among students majoring in the helping professions. Journal of Homosexuality, v.64, n.14, p.2011-2029, 2017.

ALI, Nareesa; FLEISHER, William; ERICKSON, Jueli. Psychiatrists' and psychiatry residents' attitudes toward transgender people. Academic Psychiatry, v. 40, n.2, p.268-273, 2016.

AMERICAN PSYCHOLOGICAL ASSOCIATION (APA). Guidelines for psychological practice with transgender and gender nonconforming people. American Psychologist, v. 70, n. 9, p.832-864, 2015. 
AMODEO, Anna L.; VITELLI, Roberto; SCANDURRA, Cristiano; PICARIELLO, Simona; VALERIO, Paolo. Adult attachment and transgender identity in the italian context: clinical implications and suggestions for further research. The International Journal of Transgenderism, v.16, n.1, p 49-61, 2015.

ARCELUS, Jon; LAURENCE; Witcomb, GEMMA L. Witcomb; MARSHALL, Ellen; BOUMAN, Walter Pierre. Risk factors for non-suicidal self-injury among trans youth. The Journal of Sexual Medicine, v. 13, n. 3, p.402-412, 2016.

AUSTIN, Ashley; GOODMAN, Revital. The impact of social connectedness and internalized transphobic stigma on self-esteem among transgender and gender nonconforming adults. Journal of Homosexuality, v. 64, n. 6, p.825-841, 2017.

BAUER, Greta R.; SCHEIM, Ayden I.; PYNE, Jake; TRAVERS, Robb; HAMMOND, Rebecca. Intervenable factors associated with suicide risk in transgender persons: a respondent driven sampling study in Ontario, Canada. BMC Public Health, v. 15, n. 1, p.525, 2015.

BENEDETTI, Marcos. Toda feita: o corpo e o gênero das travestis. Rio de Janeiro: Garamond, 2005.

BOLAND, Annie. God of the hinge: treating LGBTQIA patients. Journal of Analytical Psychology, v. 62, n.5, p.688-700, 2017.

BORNSTEIN, Donica R.; FAWCETT, Jake; SULLIVAN, Mirianne; SENTURIA, Kirsten D.; SHIU-THORNTON, Senturia. Understanding the experiences of lesbian, bisexual and trans survivors of domestic violence: a qualitative study. Journal of Homosexuality, v. 51, n. 1, p.159-181, 2006.

BUTLER, Judith. Problemas de gênero: feminismo e subversão da identidade. Rio de Janeiro: Civilização Brasileira, 1990/2003

BUTLER, Judith. Regulações de gênero. Caderno pagu, v. 42, p. 249-274, 2014.

BRAUN, Hannan M.; GARCIA-GROSSMAN, Ilana R.; QUIÑONES-RIVERA, Andrea; DEUTSCH, Madeline B. Outcome and impact evaluation of a transgender health course for health profession students. LGBT Health, v. 4, n. 1, p.55-61, 2017.

CALDAS, José Manuel Peixoto; FONSECA, Laura; ALMEIDA, Sofia; ALMEIDA, Lígia. Escuela y diversidad sexual: ¿que realidad?. Educação em Revista, v. 28, n. 3, p.143-158, 2012.

CALLAHAN, Matthew; ZUKOWSKI, Kyle T. Reactions to transgender women and men in public restrooms: correlates and gender differences. Journal of Homosexuality, v. 23, p.1-22, 2017.

CARRERA-FERNÁNDEZ, María Victoria; LAMEIRAS-FERNÁNDEZ, María; RODRÍGUEZ-CASTRO, Yolanda; VALLEJO-MEDINA, Pablo. Spanish adolescents' attitudes toward transpeople: proposal and validation of a short form of the Genderism and Transphobia Scale. The Journal of Sex Research, v. 51, n. 6, p.654-666, 2014.

CARROLL, Lynne; MIZOCK, Lauren. (2017). Clinical issues and affirmative treatment with trangender clients, Psychiatric clinics of north américa. Elsevier: New York, 2017.

CHAPMAN, Rose; WARDROP, Joan; FREEMAN, Phoenix; ZAPPIA, Tess; WATKINS, Rochelle; SHIELDS, Linda. A descriptive study of the experiences of lesbian, gay and transgender parents accessing health services for their children. Journal of Clinical Nursing, v.21, n.7-8, p.128-1135, 2012.

CLAES, Laurence.; BOUMAN, Walter Pierre; WITCOMB, Gemma; Thurston, Megan; FERNANDEZ-ARANDA, Fernando; ARCELUS, Jon. Non-suicidal self-injury in trans people: associations with psychological symptoms, victimization, interpersonal functioning, and perceived social support. The Journal of Sexual Medicine, v.12, n.1, p.168-179, 2015.

COSTA, Pedro Alexandre; DAVIES, Michelle. Portuguese adolescents' attitudes toward sexual minorities: transphobia, homophobia, and gender role beliefs. Journal of Homosexuality, v. 59, n. 10, p.1424-1442, 2012.

DALEY, Andrea; MACDONNELL, Judith A. Gender, sexuality and the discursive representation of access and equity in health services literature: implications for LGBT communities. International Journal for Equity in Health, v. 10, n. 40, 2011.

Periódicus, Salvador, n.11, v. 2, mai-out.2019 - Revista de estudos indisciplinares em gêneros e sexualidades Publicação periódica vinculada ao Núcleo de Pesquisa NuCuS, da Universidade Federal da Bahia - UFBA ISSN: 2358-0844 - Endereço: http://www.portalseer.ufba.br/index.php/revistaperiodicus 
DEVÍS-DEVÍS, José; PEREIRA-GARCÍA, Sofía; VALENCIA-PERIS, Alexandra; FUENTES-MIGUEL, Jorge; LÓPEZ-CAÑADA, Elena; PÉREZ-SAMANIEGO, Víctor. Harassment patterns and risk profile in spanish trans persons. Journal of Homosexuality, v. 64, n. 2, p.239-255, 2017.

ESCUDERO, Daniel J.; KERR, Thomas; OPERARIO, Don; SOCÍAS, Maria E.; SUED, Omar; MARSHALL, Brandon. D. L. Inclusion of trans women in pre-exposure prophylaxis trials: a review. AIDS Care, v. 27, n. 5, p.637-641, 2015.

FERNANDES, Felipe Bruno Martins. Assassinatos de travestis e "pais de santo" no Brasil: homofobia, transfobia e intolerância religiosa. Saúde em Debate, v. 37, n.98, p.485-492, 2013.

FISHER, Alessandra Daphne; CASTELLINI Giovanni; RISTORI, Jiska; CASALE, Helen; GIOVANARDI, Guido; CARONE, Nicola; FANNI, Egidia; MOSCONI, Maddalena; CIOCCA, Giacomo; JANNINI, Emmanuele A.; RICCA, Valdo; LINGIARDI, Vittorio; MAGGI, Mario. Who has the worst attitudes toward sexual minorities? Comparison of transphobia and homophobia levels in gender dysphoric individuals, the general population and health care providers. Journal of Endocrinological Investigation, v. 40, n. 2, p.263-273, 2017.

FRANCO, Neil; CICILLINI, Graça Aparecida. Professoras trans brasileiras em seu processo de escolarização. Revista Estudos Feministas, v. 23, n. 2, p.325-346, 2015.

HOFFKLING, Alexis; OBEDIN-MALIVER, Juno; SEVELIUS, Jae. From erasure to opportunity: a qualitative study of the experiences of transgender men around pregnancy and recommendations for providers. BMC Pregnancy and Childbirth, v. 17, n. 2, p.7-20, 2017.

HOPIA, Hanna; LATVALA, Eila; LIIMATAINEN, Leena. Reviewing the methodology of an integrative review. Scandinavian Journal of Caring Sciences, v. 30, n. 4, p.662-669, 2016.

IANTAFFI, Alex; BOCKTING, Walter O. Views from both sides of the bridge? Gender, sexual legitimacy and transgender people's experiences of relationships. Culture, Health \& Sexuality, v. 13, n.3, p.355-370, 2011.

JACKMAN, Kasey B.; DOLEZAL Curtis; BOCKTING, Walter O. Generational differences in internalized transnegativity and psychological distress among feminine spectrum transgender people. LGBT Health, v. 5, n. 1, p.54-60, 2017.

JARDIM, Juliana G. Deveriam os estudos queer falar em cis-heteronormatividade? Reflexões a partir de uma pesquisa sobre performatividade de gênero nas artes marciais mistas femininas. In \#4 Seminário Internacional de Educação e Sexualidade e \#2 Encontro Internacional de Estudos de Gênero. Vitória: Universidade Federal do Espírito Santo, 2016. Disponível em:

http://www.gepsexualidades.com.br/resources/anais/6/1467327007_ARQUIVO_Jardim _CisHeteronormatividade.pdf $>$. Acesso em 11 out 2019.

JOKIĆ-BEGIĆ, Natasa; KORAJLIJA, Anita L.; JURIN, Tanja. Psychosocial adjustment to sex reassignment surgery: a qualitative examination and personal experiences of six transsexual persons in croatia. The Scientific World Journal, v. 2014, p.960745, 2014.

KAPLAN, Rachel L.; WAGNER, Glenn J.; NEHME, Simon; AUNON, Frances; KHOURI, Danielle; Mokhbat, Jacques. Forms of safety and their impact on health: an exploration of hiv/aids-related risk and resilience among trans women in Lebanon. Health Care Women Int., v. 36, n. 8, p.917-935, 2015.

KOSENKO, Kami; RINTAMAKI, Lance; RANEY, Stephanie; MANESS, Kathlenn. Transgender patient perceptions of stigma in health care contexts. Medical Care, v. 51, n. 9, p.819-822, 2013.

LACOMBE-DUNCAN, Ashley. An intersectional perspective on access to hiv-related healthcare for transgender women. Transgender Health, v. 1, n. 1, p.137-141, 2016.

LEVITT, Nathan. Clinical nursing care for transgender patients with cancer. Clinical Journal of Oncology Nursing, v. 19, n. 3, p.362-366, 2015.

LOGIE, Carmen H.; JAMES, Llana; THARAO, Wangari; LOUTFY, Mona R. HIV, gender, race, sexual orientation, and sex work: a qualitative study of intersectional stigma experienced by HIV-positive women in Ontario, Canada. PloS Medicine, v. 8, n. 11, p. e1001124, 2011.

Periódicus, Salvador, n.11, v. 2, mai-out.2019 - Revista de estudos indisciplinares em gêneros e sexualidades Publicação periódica vinculada ao Núcleo de Pesquisa NuCuS, da Universidade Federal da Bahia - UFBA ISSN: 2358-0844 - Endereço: http://www.portalseer.ufba.br/index.php/revistaperiodicus 
LOMBARDI, Emilia. Varieties of transgender/transsexual lives and their relationship with transphobia. Journal of Homosexuality, v. 56, n. 8, p.977-99, 2011.

LOGIE, Carmen H.; JAMES, Llana; THARAO, Wangari; LOUTFY, Mona R. "We don't exist": a qualitative study of marginalization experienced by HIV-positive lesbian, bisexual, queer and transgender women in Toronto, Canada. Journal of the Internacional AIDS Society, v. 15, n. 2, p.17392, 2012.

LOVAAS, Karen E.; BAROUDI, Lina; COLLINS, Sean. M. Transcending heteronormativity in the classroom. Journal of Lesbian Studies, v. 6, n. 3-4, p.177-189, 2002.

LYONS, Tara; KRÜSI, Andrea; PIERRE, Leslie; KERR, Thomas; SMALL, Will; SHANNON, Kate. Negotiating violence in the context of transphobia and criminalization: the experiences of trans sex workers in Vancouver, Canada, Qualitative Health Research. v.27, n.2, p. 182-190, 2017.

LYONS, Tara; SHANNON, Kate; PIERRE, Lesline; SMALL, Will; KRÜSI, Andrea; KERR, Thomas. A qualitative study of transgender individuals' experiences in residential addiction treatment settings: stigma and inclusivity. Substance Abuse Treatment, Prevention, and Policy, v. 10, n.17, 2015.

MCDERMOTT, Elizabeth; HUGHES, Elizabeth; RAWLINGS, Victoria. The social determinants of lesbian, gay, bisexual and transgender youth suicidality in England: a mixed methods study. Journal of Public Health. v. 17, p.1-8, 2017.

MCPHAIL, Deborah; ROUNTREE-JAMES, Marina; WHETTER, Ian. Addressing gaps in physician knowledge regarding transgender health and healthcare through medical education. Candian Medical Education Journal, v. 7, n. 2, p.e70-e78, 2016.

MOTT, Luiz. Homo-afetividade e direitos humanos. Revista Estudos Feministas, v.14, n.2, p. 509-521, 2006.

MÜLLER, Alex. Scrambling for access: availability, accessibility, acceptability and quality of healthcare for lesbian, gay, bisexual and transgender people in South Africa. BMC International Health and Human Rights, v.17, n.16, 2017.

MUÑOZ-LABOY, Miguel; SEVERSON, Nicolette; LEVINE, Ethan; MARTÍNEZ, Omar. Latino men who have sex with transgender women: the influence of heteronormativity, homonegativity and transphobia on gender and sexual scripts. Culture, Health \& Sexuality, v. 19, n.9, p.964-978, 2017.

NEMOTO, Tooru; BÖDEKER, Birte; IWAMOTO, Mariko. Social support, exposure to violence and transphobia, and correlates of depression among male-to-female transgender women with a history of sex work. American Public Health Association, v. 101, n.10, p.19801988, 2011.

NEMOTO, Tooru; IWAMOTO, Mariko; PERNGPARN, Usaneya; AREESANTICHAI, Chitlada; KAMITANI, Emiko; SAKATA, Maria. HIV-related risk behaviors among kathoey (male-to-female transgender) sex workers in Bangkok, Thailand. AIDS Care, v. 24, n. 2, p.210-219, 2012.

NEMOTO, Toru; OPERARIO, Don; KEATLEY, Joanne; VILLEGAS, Dolors. Social context of HIV risk behaviours among male-to-female transgenders of colour. AIDS Care, v. 16, n. 6, p.724-735, 2004.

NEWMAN-VALENTINE, Douglas; DUMA, Sinegugu. Injustice to transsexual women in a hetero-normative healthcare system. African Journal of Primary Health Care \& Family Medicine, v.6, n.1, p.1-5, 2014.

O'NEILL, Thomas; WAKEFIELD, Justin. Fifteen-minute consultation in the normal child: Challenges relating to sexuality and gender identity in children and young people. Archives of Disease in Childhood Education \& Practice, v. 102, n. 6, p.298-303, 2017.

ORGANIZAÇÃO DAS NAÇÕES UNIDAS (ONU). Promoção dos direitos humanos de pessoas LGBT no mundo do trabalho. Projeto "construindo a igualdade de oportunidades no mundo do trabalho: combatendo a homo-lesmo-transfobia". PNUD, OIT, UNAIDS. 2014. Disponível em: < https://unaids.org.br/wpcontent/uploads/2016/01/2015_ManualPromocaoDireitosLGBTTrabalho_PT_V2.pdf >. Acesso em 11 out 2019.

Periódicus, Salvador, n.11, v. 2, mai-out.2019 - Revista de estudos indisciplinares em gêneros e sexualidades Publicação periódica vinculada ao Núcleo de Pesquisa NuCuS, da Universidade Federal da Bahia - UFBA ISSN: 2358-0844 - Endereço: http://www.portalseer.ufba.br/index.php/revistaperiodicus 
ORGANIZAÇÃO DAS NAÇÕES UNIDAS PARA A EDUCAÇÃO, A CIÊNCIA E A CULTURA (UNESCO). Jogo Aberto: respostas do setor de educação à violência com base na orientação sexual e na identidade/expressão de gênero. Relatório Conciso. 2017. Disponível em: <http://unesdoc.unesco.org/images/0024/002446/244652POR.pdf>. Acesso em 11 out 2019.

OTERO, Luis Manuel Rodríguez; FERNÁNDEZ, Maria Victoria Carrera; FERNÁNDEZ, Maria Lameiras; CASTRO, Yolanda Rodríguez. Violencia en parejas transexuales, transgénero e intersexuales: una revisión bibliográfica. Saúde e Sociedade, v. 24, n. 3, p.914935, 2015.

PENNINGTON, Jarred; KNIGHT, Tess. Through the lens of hetero-normative assumptions: Re-thinking attitudes towards gay parenting. Culture, Health \& Sexuality, v. 13, n.1, p.59-72, 2011.

PEREZ-BRUMER, Amaya; HATZENBUEHLER, Mark L.; OLDENBURG, Catherine E.; BOCKTING, Walter. Individual and structural-level risk factors for suicide attempts among transgender adults. Behavioral Medicine, v. 41, n. 3, p.164-171, 2015.

ROCON, Pablo Cardozo; RODRIGUES, Alexsandro; ZAMBONI, Jésio; PEDRINI, Mateus Dias. Dificuldades vividas por pessoas trans no acesso ao Sistema Único de Saúde. Ciência \& Saúde Coletiva, v. 21, n. 8, p.2517-2526, 2016.

SAUSA, Lydia A.; KEATLEY, Joanne; OPERARIO, Don. Perceived risks and benefits of sex work among transgender women of color in San Francisco. Archives of Sexual Behavior, v. 36, n. 6, p.768-777, 2007.

SEVELIUS, Jae M.; KEATLEY, Joanne; CALMA, Nikki; ARNOLD, Emily. 'I am not a man': Trans-specific barriers and facilitators to PrEP acceptability among transgender women. Global Public Health, v. 11, n. 7-8, p.1060-1075, 2016.

SOUZA, Marcela Tavares; SILVA, Michelly Dias; CARVALHO Rachel. Revisão integrativa: o que é e como fazer. Einstein (São Paulo), v. 8, n. 1, p.102-106, 2010.

STAPLES, Jennifer M; NEILSON, Elizabeth C.; BRYAN, Aamanda E. B.; GEORGE, William $\mathrm{H}$. The role of distal minority stress and internalized transnegativity in suicidal ideation and nonsuicidal self-injury among transgender adults. The Journal of Sex Research, v. 55 , n. 4-5, p.1-13, 2017.

SUGANO, Eiko; NEMOTO, Tooru; OPERARIO, Don. The impact of exposure to transphobia on HIV risk behavior in a sample of transgendered women of color in San Francisco. AIDS and Behavior, v. 10, n. 2, p.217-225, 2006.

TAYLOR, Catharine; PETER, Tracey. "We are not aliens, we're people, and we have rights." Canadian human rights discourse and high school climate for LGBTQ students. Canadian Review of Sociology, v. 48, n. 3, p.275-312, 2011.

TEBBE, Esther N.; BONNIE, Moradi. Anti-trangender prejudice: a structural equation model of associated constructs. Journal of Counseling Psychology, v. 59, n. 2, p.251-261, 2012.

TESTA, Rylan J.; MICHAELS, Michaels S.; BLISS, Whitney; ROGERS, Megan L.; BALSAM, Kimb F.; JOINER, Thomas. Suicidal ideation in transgender people: gender minority stress and interpersonal theory factors. Journal of Abnormal Psychology, v. 126, n. 1, p.125-136, 2017.

WEINER, Brittany A.; ZINNER, Leah. Attitudes toward straight, gay male, and transsexual parenting. Journal of Homosexuality, v. 62, n. 3, p.327-339, 2015.

WHITTEMORE, Robin; KNAFL, Kathleen. The integrative review: updated methodology. Journal of Advanced Nursing, v. 52, n.5, p.546-553, 2005.

VERGUEIRO, Viviane. Por inflexões decoloniais de corpos e identidades de gênero inconformes: uma análise autoetnográfica da cisgeneridade como normatividade. 2015. 243fs. Dissertação (Programa Multidisciplinar de Pós-Graduação em Cultura e Sociedade) - Universidade Federal da Bahia, Salvador, 2015.

ZERBINATI, João Paulo; BRUNS, Maria Alves de Toledo. Transidentidade: da ruptura patologizante ao matiz da criatividade. Curitiba: CRV, 2018.

Periódicus, Salvador, n.11, v. 2, mai-out.2019 - Revista de estudos indisciplinares em gêneros e sexualidades Publicação periódica vinculada ao Núcleo de Pesquisa NuCuS, da Universidade Federal da Bahia - UFBA ISSN: 2358-0844 - Endereço: http://www.portalseer.ufba.br/index.php/revistaperiodicus 